\title{
Modeling and Frequency Performance Analysis of Through Silicon Capacitors in Silicon Interposers
}

\author{
Khadim Dieng, Philippe Artillan, Cédric Bermond, Olivier Guiller, Thierry Lacrevaz, Sylvain Joblot, \\ Grégory Houzet, Alexis Farcy, Anne-Laure Perrier, Yann Lamy, and Bernard Fléchet
}

\begin{abstract}
The feasibility of cointegration of new capacitors, named "through silicon capacitors" (TSCs) with "through silicon vias" in silicon interposers has recently been demonstrated. Two architectures of TSC are extensively investigated in this paper: "axial TSC" whose electrodes are connected on either sides of the silicon interposer and "radial TSC" with electrodes both connected to the metal layers of the back end of line. A general modeling method based on distributed cell segmentation is proposed for both architectures. Validation is performed by measurements from $1 \mathrm{kHz}$ to $40 \mathrm{GHz}$ (above the resonance frequency of the components). A comparative study between radial and axial architectures is performed, leading to the prediction of the performances of those new components. Finally, design rules are established for future integration for power delivery networks decoupling applications.
\end{abstract}

Index Terms - High frequency modeling, integrated capacitor, measurements, power distribution network (PDN), silicon interposer.

\section{INTRODUCTION}

$\mathbf{T}$ HE integration of high-value and high-frequency decoupling capacitors for power distribution network (PDN) applications in 3-D integrated circuits still remains a technological requirement [1]. As decoupling capacitors must be placed at the very close vicinity of the integrated devices, embedded MIM capacitors have been extensively used [2], [3]. However, since the overall dedicated area for the capacitors is constantly reduced, while capacitance value needs to be increased, their fabrication process has been improved in many ways (insulator thickness decrease, dielectric material permittivity enhancement). Those improvements are probably now reaching their limits [4], [5]. Furthermore, 2.5-D integration, with silicon interposers, provides alternative locations for efficient, high value and high density 3-D capacitors. Inspired from the through silicon vias (TSV), a very promising technology seems to be the "through silicon capacitors" (TSCs), i.e., vertical cylindrical capacitors embedded through the silicon interposer. They are investigated in [6] and present

Manuscript received July 25, 2016; revised November 28, 2016; accepted January 11, 2017. Date of publication February 13, 2017; date of current version April 18, 2017. This work was supported by ARC6, Région Auvergne-Rhône-Alpes, France. Recommended for publication by Associate Editor S. Grivet-Talocia upon evaluation of reviewers' comments.

K. Dieng, P. Artillan, C. Bermond, T. Lacrevaz, G. Houzet, A.-L. Perrier, and B. Fléchet are with Université Savoie Mont-Blanc, IMEP-LAHC, 73376 Le Bourget du Lac, France (e-mail: khadim.dieng@univ-smb.fr; philippe.artillan@univ-smb.fr; cedric.bermond@univ-smb.fr).

O. Guiller, S. Joblot, and A. Farcy are with STMicroelectronics, 38926 Crolles, France.

Y. Lamy is with Université Grenoble Alpes, 38000 Grenoble, France, and also with CEA, LETI, 38054 Grenoble, France.

Digital Object Identifier 10.1109/TCPMT.2017.2655939 high capacitance density (up to $35 \mathrm{nF} / \mathrm{mm}^{2}$ ). High capacitance values are given by the use of a large amount of TSCs connected in parallel as a matrix. Typical diameter of a TSC is $10 \mu \mathrm{m}$, and depth is $100 \mu \mathrm{m}$. A matrix can contain thousands of TSCs in parallel. On the top and bottom sides of the silicon interposer, metal layers (called lids) are used to connect them in parallel, and they present associated parasitic elements (for inductive and resistive effects) that limit the decoupling function. The parasitic effects have then to be modeled and measured from dc to several gigahertz (above the resonance frequency of the components) [7].

Using standard full-wave finite element analysis (FEA) software to model complete structure of hundreds of TSCs would necessitate huge resources and computing time, and such simulations seem to be unreachable in some cases. To overcome this issue and to be able to quantify the parasitic elements of large matrices of TSC, one developed two modeling methods for two architectures of TSC [8]. Those modeling methods, based on a segmented approach, make use of 2-D/3-D parasitic extraction software for the modeling of each part of the structure. The resulting lumped RLCG parameters are used to generate a global equivalent circuit composed of segments of coupled distributed cells. Section II gives an overview of the technological process for the integration of TSC. Section III presents the modeling methods for each architecture. Section IV provides a comparative study of those two architectures, with further investigation on the impact of the number of TSCs and the excitation.

\section{Technological Realization of Axial AND RADIAL TSC MATRICES}

TSCs are copper vertical cylindrical capacitors inspired from the TSV. The TSC technology is based on an MIM stack deposited in deep holes etched in a silicon interposer. Two kinds of structures are investigated in this paper: axial and radial structures; as described in Fig. 1. In the axial structure, the inner conductors of each TSC are connected together by a metal layer on the top side of the interposer, and the outer conductors are connected on the back side. On the contrary, in the radial structure, both the inner and the outer conductors are connected on the top side of the interposer.

The presented demonstrators have been realized using the radial process, as shown in Fig. 2. Matrices of deep holes have been filled with a standard capacitive stack $\operatorname{TiN}(200 \mathrm{~nm}) / \mathrm{Al}_{2} \mathrm{O}_{3}(20 \mathrm{~nm}) / \mathrm{TiN}(200 \mathrm{~nm})$ as shown in Fig. 3. The integration of TSC shares many common steps with the integration of TSV: deep reactive ion etching, passivation, and copper filling. This cointegration with TSV leads to 


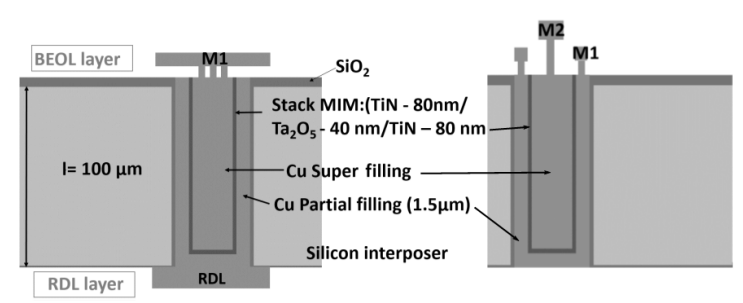

Axial TSC

Radial TSC

Fig. 1. Radial and axial TSC architectures.

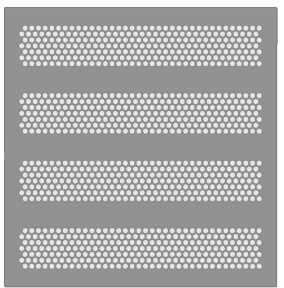

(a)

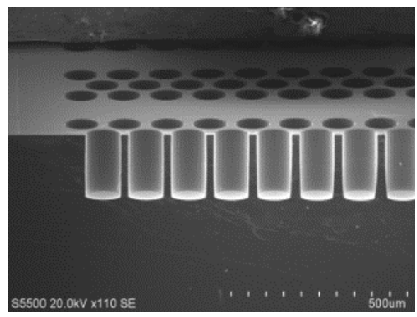

(b)
Fig. 2. (a) Top view of a matrix of 1008 TSC (diameter $100 \mu \mathrm{m}$, depth $188 \mu \mathrm{m}$ ). (b) Tilted SEM cross section of TSC matrix (CEA LETI).

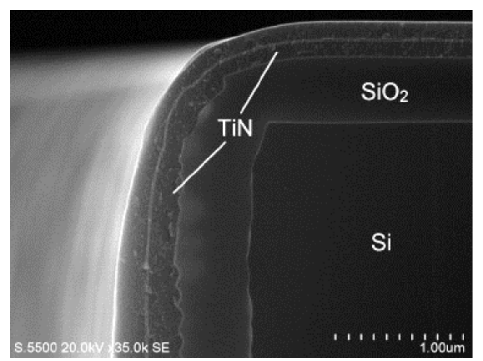

Fig. 3. SEM cross section of a $\mathrm{SiO}_{2} / \mathrm{TiN} / \mathrm{Al}_{2} \mathrm{O}_{3} / \mathrm{TiN}$ stack deposited in a deep hole (CEA LETI).

an overall cost reduction of the process. The full fabrication process is detailed in [9]. These first demonstrators are intended to prove the technological feasibility of TSC components and to evaluate their performances in terms of capacitance density, parasitic resistance, and parasitic inductance compared to planar MIM components.

\section{Modeling Method For Axial And RAdial TSCS}

Numerous TSC coaxial bodies connected in parallel by lids constitute a matrix. Capacitances much higher than $10 \mathrm{nF}$ for decoupling applications can be achieved by large matrices of TSCs (more than 1000 TSCs), but the full simulation of such matrices still remains a challenge due to the number of elements and to their aspect ratio. The two modeling methods developed in this paper allow the estimation of the performances of large matrices of TSCs. The obtained frequency response is valid up to a decade above the serial resonance frequency (SRF) as all electromagnetic effects are taken into account, i.e., inductive and resistive parasites in conductors and capacitive effect as well as conductive losses in dielectrics. The decoupling of electrical and magnetic simulations considerably reduces the computing time, thus allowing the simulation of very large matrices that are unreachable with standard full-wave FEA software.
The first modeling method, described in part A, is dedicated to "axial TSC," whereas the second modeling method, described in part B, is dedicated to "radial TSC." The two topologies are presented in Fig. 1. For both topologies, the whole component is composed of horizontal metal plates, named "lids," and vertical coaxial cylindrical electrodes, named TSC bodies. Both methods consider that the entire structure can be considered as cascaded subcircuits under the assumption that there is no coupling between each subcircuit. The inductive coupling between lids and TSC bodies is null since the current densities are perpendicular one to another. The capacitive and conductive coupling is negligible, except in axial case, where it has been modeled analytically with $\mathrm{Z}$ coupling (see Fig. 4).

\section{A. Axial TSC}

The structural electrical circuit equivalent to the axial architecture is presented in Fig. 4. The full component is composed of five main subcircuits: the coaxial bodies, the top and bottom lids, and two parallel connection stages. The principle of the modeling method is to determine separately the ABCD matrix of each subcircuit (see Sections III-A.1 and III-A.2) and to take the matrix product of them, then to use the admittance representation to make the parallel connections and finally obtain the equivalent impedance of the system (see Section III-A.3).

1) TSC Coaxial Bodies Contribution: The $N$ coaxial TSC bodies can be viewed as $2 N$ coupled transmission lines ( $N$ inner conductors and $N$ outer conductors). The departure point of the proposed approach is that no conductor is considered as a "return path," and that a virtual ground conductor is therefore defined.

As it is based on the resolution of field integral equations, the magnetic 3-D solver of Ansys Q3D Extractor allows the resolution of magneto-quasi-static problems including portions of loops of currents, thus leading to the evaluation of partial inductances. In the same manner, the electric 3-D solver allows the resolution of electro-quasi-static problems including voltage drops between conductors in partial influence without defined ground, thus leading to the evaluation of partial capacitances. The losses are also included in simulations, and the output matrices of the two solvers are then $\boldsymbol{Z}_{\boldsymbol{M}}$ $(2 N \times 2 N$ dense matrix modeling the inductive and resistive coupled effects in the $2 N$ conductors $)$ and $\boldsymbol{Y}_{\boldsymbol{E}}(2 N \times 2 N$ dense matrix modeling the capacitive and conductive effects between the $2 N$ conductors). For the sake of clearness, vector and matrix names have been bolded.

Given $\boldsymbol{Z}_{\boldsymbol{M}}$ and $\boldsymbol{Y}_{\boldsymbol{E}}$, one can compute the generalized ABCD matrix, named $\boldsymbol{A}_{B O D I E S}$, of the coaxial TSC bodies, thanks to the method presented in [10]

$$
\left[\begin{array}{c}
\boldsymbol{V}_{\boldsymbol{A}} \\
\boldsymbol{V}_{\boldsymbol{B}} \\
\boldsymbol{I}_{\boldsymbol{A}} \\
\boldsymbol{I}_{\boldsymbol{B}}
\end{array}\right]=\underbrace{\left[\begin{array}{cc}
\boldsymbol{Y}_{\boldsymbol{E}}^{-1} \cdot \operatorname{coshm}(\boldsymbol{\Gamma}) \cdot \boldsymbol{Y}_{\boldsymbol{E}} & \boldsymbol{Z}_{\boldsymbol{C}} \cdot \operatorname{sinhm}(\boldsymbol{\Gamma}) \\
\operatorname{sinhm}(\boldsymbol{\Gamma}) \cdot \boldsymbol{Z}_{\boldsymbol{C}}^{-1} & \operatorname{coshm}(\boldsymbol{\Gamma})
\end{array}\right]}_{\boldsymbol{A}_{B O D I E S}}\left[\begin{array}{c}
\boldsymbol{V}_{\boldsymbol{C}} \\
\boldsymbol{V}_{\boldsymbol{D}} \\
-\boldsymbol{I}_{\boldsymbol{C}} \\
-\boldsymbol{I}_{\boldsymbol{D}}
\end{array}\right]
$$

where

$$
\begin{gathered}
\boldsymbol{\Gamma}=\operatorname{sqrtm}\left(\boldsymbol{Y}_{\boldsymbol{E}} \cdot \boldsymbol{Z}_{\boldsymbol{M}}\right) \\
\boldsymbol{Z}_{\boldsymbol{c}}=\operatorname{sqrtm}\left(\boldsymbol{Y}_{\boldsymbol{E}}^{-1} \cdot \boldsymbol{Z}_{\boldsymbol{M}}\right)
\end{gathered}
$$




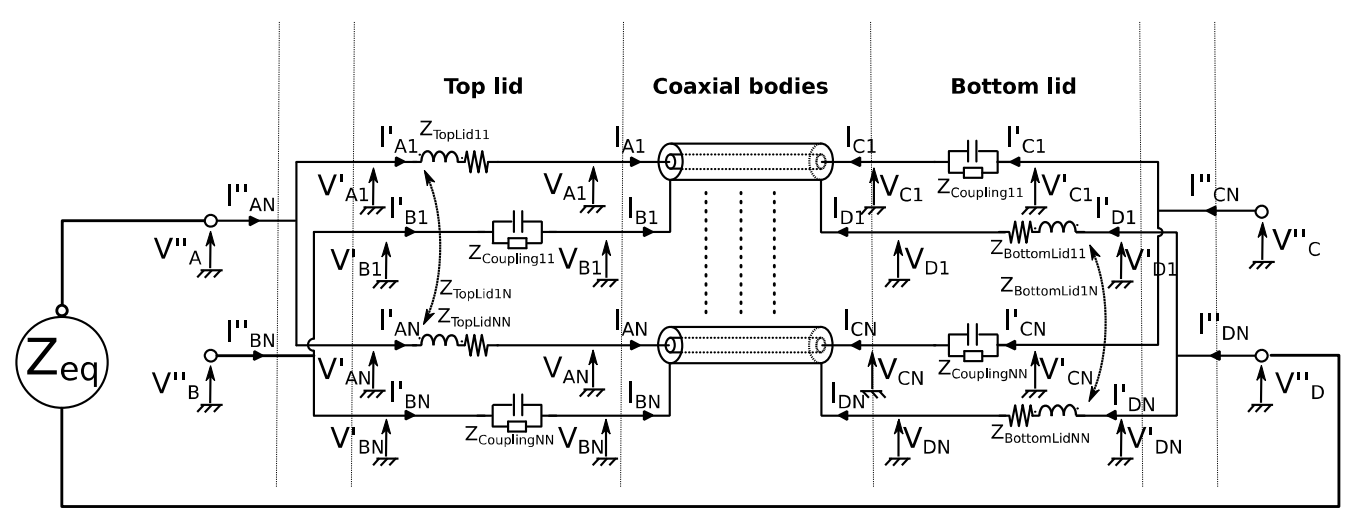

AXIAL TSC

Fig. 4. Axial TSC structural electrical model.

and where the voltage and current vectors of the TSC coaxial bodies

$$
\begin{gathered}
\boldsymbol{V}_{\boldsymbol{A}}=\left[\begin{array}{c}
V_{A 1} \\
\vdots \\
V_{A N}
\end{array}\right] \quad \boldsymbol{I}_{\boldsymbol{A}}=\left[\begin{array}{c}
I_{A 1} \\
\vdots \\
I_{A N}
\end{array}\right] \quad \boldsymbol{V}_{\boldsymbol{B}}=\left[\begin{array}{c}
V_{B 1} \\
\vdots \\
V_{B N}
\end{array}\right] \\
\boldsymbol{I}_{\boldsymbol{B}}=\left[\begin{array}{c}
I_{B 1} \\
\vdots \\
I_{B N}
\end{array}\right] \quad \boldsymbol{V}_{\boldsymbol{C}}=\left[\begin{array}{c}
V_{C 1} \\
\vdots \\
V_{C N}
\end{array}\right] \quad \boldsymbol{I}_{\boldsymbol{C}}=\left[\begin{array}{c}
I_{C 1} \\
\vdots \\
I_{C N}
\end{array}\right] \\
\boldsymbol{V}_{\boldsymbol{D}}=\left[\begin{array}{c}
V_{D 1} \\
\vdots \\
V_{D N}
\end{array}\right] \quad \text { and } \boldsymbol{I}_{\boldsymbol{D}}=\left[\begin{array}{c}
I_{D 1} \\
\vdots \\
I_{D N}
\end{array}\right]
\end{gathered}
$$

are defined in Fig. 4. Furthermore, sqrtm, sinhm, and coshm, respectively, denote the matrix square root, matrix hyperbolic cosine, and matrix hyperbolic sine functions. It is interesting to note that the simulation directly gives the extensive matrix $\boldsymbol{\Gamma}$, i.e., the equivalent line length does not need to be known as it is included in $\Gamma$.

2) Top and Bottom Lids Contribution: The ABCD matrices $\boldsymbol{A}_{\text {Tор }}\left(\boldsymbol{A}_{\text {Bоттом }}\right)$ of the top lid (bottom lid) are computed thanks to the following:

1) the magnetic simulation of the inductive effects in the lids that provides $\boldsymbol{Z}_{\text {TopLid }}\left(\boldsymbol{Z}_{\text {BottomLid }}\right)$;

2) the analytical evaluation of the coupling effect between the TSC coaxial body and the lid that lid to the highimpedance diagonal matrix $\boldsymbol{Z}_{\text {Coupling }}$

$$
\begin{aligned}
& {\left[\begin{array}{c}
\boldsymbol{V}_{\boldsymbol{A}}^{\prime} \\
\boldsymbol{V}_{\boldsymbol{B}}^{\prime} \\
\boldsymbol{I}_{\boldsymbol{A}}^{\prime} \\
\boldsymbol{I}_{\boldsymbol{B}}^{\prime}
\end{array}\right]=\underbrace{\left[\begin{array}{cccc}
\mathbb{I}_{N} & \mathbb{O}_{N} & \boldsymbol{Z}_{\text {TopLid }} & \mathbb{O}_{N} \\
\mathbb{O}_{N} & \mathbb{I}_{N} & \mathbb{O}_{N} & \boldsymbol{Z}_{\text {Coupling }} \\
\mathbb{O}_{N} & \mathbb{O}_{N} & \mathbb{I}_{N} & \mathbb{O}_{N} \\
\mathbb{O}_{N} & \mathbb{O}_{N} & \mathbb{O}_{N} & \mathbb{I}_{N}
\end{array}\right]}_{\boldsymbol{A}_{\text {TOP }}} \cdot\left[\begin{array}{c}
\boldsymbol{V}_{\boldsymbol{A}} \\
\boldsymbol{V}_{\boldsymbol{B}} \\
\boldsymbol{I}_{\boldsymbol{A}} \\
\boldsymbol{I}_{\boldsymbol{B}}
\end{array}\right]} \\
& {\left[\begin{array}{c}
\boldsymbol{V}_{\boldsymbol{C}} \\
\boldsymbol{V}_{\boldsymbol{D}} \\
-\boldsymbol{I}_{\boldsymbol{C}} \\
-\boldsymbol{I}_{\boldsymbol{D}}
\end{array}\right]=\underbrace{\left[\begin{array}{cccc}
\mathbb{I}_{N} & \mathbb{O}_{N} & \boldsymbol{Z}_{\text {Coupling }} & \mathbb{O}_{N} \\
\mathbb{O}_{N} & \mathbb{I}_{N} & \mathbb{O}_{N} & \boldsymbol{Z}_{\text {BottomLid }} \\
\mathbb{O}_{N} & \mathbb{O}_{N} & \mathbb{I}_{N} & \mathbb{O}_{N} \\
\mathbb{O}_{N} & \mathbb{O}_{N} & \mathbb{O}_{N} & \mathbb{I}_{N}
\end{array}\right]}_{\boldsymbol{A}_{\text {Воттом }}} \cdot\left[\begin{array}{c}
\boldsymbol{V}_{\boldsymbol{C}}^{\prime} \\
\boldsymbol{V}_{\boldsymbol{D}}^{\prime} \\
-\boldsymbol{I}_{\boldsymbol{C}}^{\prime} \\
-\boldsymbol{I}_{\boldsymbol{D}}^{\prime}
\end{array}\right]}
\end{aligned}
$$

where $\mathbb{I}_{N}$ is the $N$-D identity matrix and $\mathbb{O}_{N}$ the $N$-D null matrix.
3) Equivalent Impedance: Once we have determined the $\mathrm{ABCD}$ matrices of the TSC bodies and of the lids, the ABCD matrix of the $N$ TSC component is given by

$$
\boldsymbol{A}_{N T S C}=\boldsymbol{A}_{T O P} \cdot \boldsymbol{A}_{\text {BODIES }} \cdot \boldsymbol{A}_{\text {BOTTOM }} .
$$

Defining $\boldsymbol{Y}_{N T S C}$ such as $\boldsymbol{I}^{\prime}=\boldsymbol{Y}_{N T S C} \cdot \boldsymbol{V}^{\prime}$, one can obtain $\boldsymbol{Y}_{N T S C}$ from $\boldsymbol{A}_{N T S C}$ using the well-known circuit matrix conversion relations [11].

In order to connect the four sets of branches A, B, C, and D in parallel as shown in Fig. 4, one defines the incidence matrix $\boldsymbol{P}$

$$
\boldsymbol{P}=\left[\begin{array}{ccccccccccccc}
1 & \cdots & 1 & 0 & & & & \cdots & & & & 0 \\
0 & \cdots & 0 & 1 & \cdots & 1 & 0 & & & \cdots & & 0 \\
0 & & \cdots & & & 0 & 1 & \cdots & 1 & 0 & \cdots & 0 \\
0 & & \cdots & & \cdots & & & \cdots & & 1 & \cdots & 1
\end{array}\right]
$$

Kirchhoff's circuit laws at nodes A, B, C, and D imply $\boldsymbol{I}^{\prime \prime}=\mathbf{P I}^{\prime}$ and $\boldsymbol{V}^{\prime}=\boldsymbol{P}^{T} \boldsymbol{V}^{\prime \prime}$, with $\boldsymbol{P}^{T}$ the transpose of $\boldsymbol{P}$. Furthermore, defining the admittance matrix $\boldsymbol{Y}_{\boldsymbol{R}}$ such as $I^{\prime \prime}=Y_{R} V^{\prime \prime}$, one can establish the following relation for the computation of $\boldsymbol{Y}_{\boldsymbol{R}}$ :

$$
\boldsymbol{Y}_{\boldsymbol{R}}=\boldsymbol{P} \cdot \boldsymbol{Y}_{N T S C} \cdot \boldsymbol{P}^{T} .
$$

The elements of this resulting reduced matrix $\boldsymbol{Y}_{\boldsymbol{R}}$ are then permuted in order to obtain the admittance $\widetilde{\boldsymbol{Y}_{\boldsymbol{R}}}$ defined between the points $\mathrm{A}$ and $\mathrm{D}$. This permutation transforms the current vector $\boldsymbol{I}^{\prime \prime}$ into $\widetilde{\boldsymbol{I}^{\prime \prime}}$ and the voltage vector $\boldsymbol{V}^{\prime \prime}$ into $\widetilde{\boldsymbol{V}^{\prime \prime}}$

$$
\begin{aligned}
\boldsymbol{I}^{\prime \prime} & =\left[\begin{array}{l}
I^{\prime \prime}{ }_{A} \\
I^{\prime \prime}{ }_{B} \\
I^{\prime \prime}{ }_{C} \\
I^{\prime \prime}{ }_{D}
\end{array}\right] \boldsymbol{V}^{\prime \prime}=\left[\begin{array}{l}
V^{\prime \prime}{ }_{A} \\
V^{\prime \prime}{ }_{B} \\
V^{\prime \prime}{ }_{C} \\
V^{\prime \prime}{ }_{D}
\end{array}\right] \widetilde{\boldsymbol{I}^{\prime \prime}}=\left[\begin{array}{c}
I^{\prime \prime}{ }_{A} \\
I^{\prime \prime}{ }_{D} \\
I^{\prime \prime}{ }_{B} \\
I^{\prime \prime}{ }_{C}
\end{array}\right] \\
\widetilde{V^{\prime \prime}} & =\left[\begin{array}{l}
V^{\prime \prime}{ }_{A} \\
V^{\prime \prime}{ }_{D} \\
V^{\prime \prime}{ }_{B} \\
V^{\prime \prime}{ }_{C}
\end{array}\right] .
\end{aligned}
$$

Defining the permutation matrix $\boldsymbol{B}$ as

$$
\boldsymbol{B}=\left[\begin{array}{llll}
1 & 0 & 0 & 0 \\
0 & 0 & 0 & 1 \\
0 & 1 & 0 & 0 \\
0 & 0 & 1 & 0
\end{array}\right]
$$




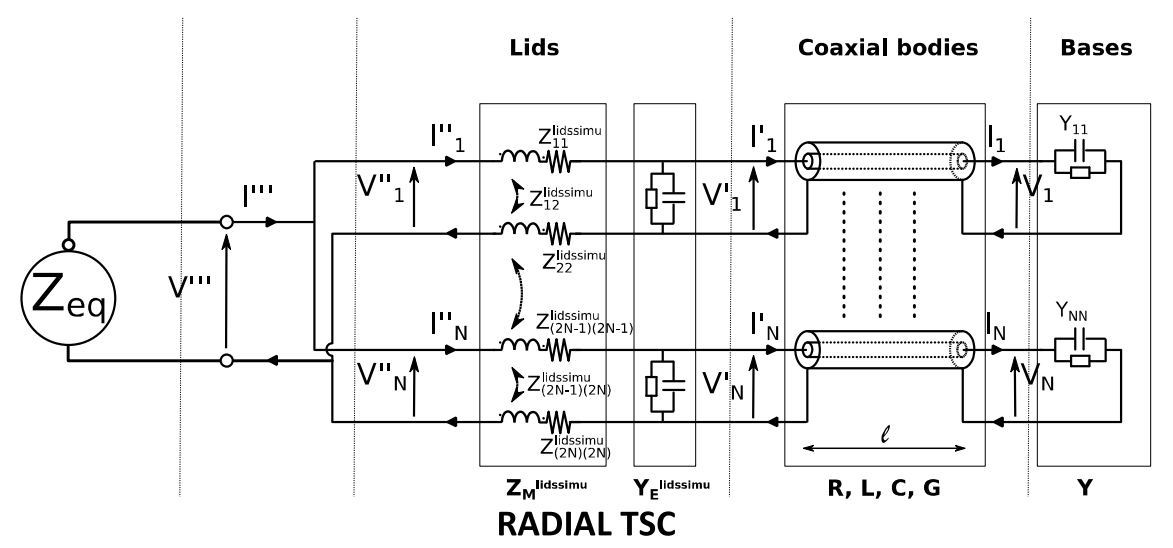

Fig. 5. Radial TSC structural electrical model.

one can relate old and new currents and voltages by writing, respectively, $\widetilde{\boldsymbol{I}^{\prime \prime}}=\boldsymbol{B} \cdot \boldsymbol{I}^{\prime \prime}$ and $\widetilde{\boldsymbol{V}^{\prime \prime}}=\boldsymbol{B} \cdot \boldsymbol{V}^{\prime \prime}$. The admittance matrix becomes, after permutation

$$
\widetilde{Y_{R}}=B \cdot Y_{R} \cdot B^{-1} \text {. }
$$

The matrix $\widetilde{\boldsymbol{Y}_{\boldsymbol{R}}}$ is then divided into four blocks (of dimension $2 \times 2$ ) $\widetilde{Y_{R_{I}}}, \widetilde{Y_{R_{I I}}}, \widetilde{Y_{R_{I I I}}}$, and ${\widetilde{Y_{R_{I V}}}}$ as follows:

$$
\widetilde{Y_{\boldsymbol{R}}}=\left[\begin{array}{cccc}
\widetilde{Y}_{R_{11}} & \widetilde{Y}_{R_{12}} & \widetilde{Y}_{R_{13}} & \widetilde{Y}_{R_{14}} \\
\widetilde{Y}_{R_{21}} & \widetilde{Y}_{R_{22}} & \widetilde{Y}_{R_{23}} & \widetilde{Y}_{R_{24}} \\
\widetilde{Y}_{R_{31}} & \widetilde{Y}_{R_{32}} & \widetilde{Y}_{R_{33}} & \widetilde{Y}_{R_{34}} \\
\widetilde{Y}_{R_{41}} & \widetilde{Y}_{R_{42}} & \widetilde{Y}_{R_{43}} & \widetilde{Y}_{R_{44}}
\end{array}\right]=\left[\begin{array}{ccc}
\widetilde{Y}_{\boldsymbol{R}_{I}} & {\widetilde{Y_{R_{I I}}}}_{\widetilde{Y_{R_{I I I}}}} & {\widetilde{Y_{R_{I V}}}}
\end{array}\right] .
$$

The $(2 \times 2)$ input impedance matrix $Z_{\text {in }}$ defined between $\mathrm{A}$ and $\mathrm{D}$ is given by

$$
Z_{\text {in }}=\left({\widetilde{Y_{R_{I}}}}-{\widetilde{Y_{R_{I I}}}} \cdot{\widetilde{Y_{R_{I V}}}}^{-1} \cdot{\widetilde{Y_{R_{I I I}}}}^{-1}\right.
$$

Finally, the equivalent scalar impedance $Z_{\text {eq }}$ of the whole TSC matrix is the off-diagonal element of $\boldsymbol{Z}_{\text {in }}$

$$
Z_{\text {eq }}=Z_{\text {in }_{12}} \text {. }
$$

\section{B. Radial TSC}

Considering a matrix of radial TSCs, one can describe the one-port capacitive component as two parallel metallic conductors on the top side of the interposer, named lids, separated by a very thin dielectric layer on which the coaxial bodies of the TSCs are connected in parallel. The bottom end of the coaxial bodies consists in capacitive bases. Fig. 5 presents the structural equivalent electrical circuit of such devices.

The principle of the modeling method for radial TSC is to determine the admittance matrix of the whole set of bases (see Section III-B.1) and to compute the resulting input admittance matrix of the device, stage after stage (coaxial bodies in Section III-B.2, then lids in Section III-B.3). Each segment is then considered as coupled distributed cells analog to coupled transmission lines, and a matrix formula derived from [10] is proposed. In the following development, the notations are the ones presented in Fig. 5.
1) TSC Bases Contribution: For the considered components, an analytic formulation of the admittance of the back side of the TSCs has turned out to be sufficient. Considering the real permittivity $\varepsilon^{\prime}$ and the conductivity $\sigma$ of the dielectric material, the equivalent admittance $\boldsymbol{Y}=\boldsymbol{I} \boldsymbol{V}^{-1}$ of the back side of a single TSC can be written as

$$
\boldsymbol{Y}=j \omega \frac{\left(\varepsilon^{\prime}-j \frac{\sigma}{\omega}\right) S}{t} \mathbb{I}_{N}
$$

where $S$ is the surface of parallel metallic plates of the back side, $t$ the distance between them, and $\mathbb{I}_{N}$ the identity matrix of dimension $N \times N$.

2) TSC Coaxial Bodies Contribution: The coaxial bodies can be considered as (weakly) coupled coaxial lines in the case of radial TSC because the inner conductor and the outer conductor are flowed by opposite currents. Their contribution, the primary matrix parameters of coupled lines $(\boldsymbol{R}, \boldsymbol{L}, \boldsymbol{C}, \boldsymbol{G})$, can thus be easily computed with a $2-\mathrm{D}$ electromagnetic solver, which is very computing-time effective. One then defines the "magnetic" impedance matrix $Z_{M}^{\text {bodies }}$ and the "electrical" admittance matrix $\boldsymbol{Y}_{\boldsymbol{E}}^{\text {bodies }}$ as follows:

$$
\begin{aligned}
& \boldsymbol{Z}_{\boldsymbol{M}}^{\text {bodies }}=\boldsymbol{R} l+j \omega \boldsymbol{L} l \\
& \boldsymbol{Y}_{\boldsymbol{E}}^{\text {bodies }}=\boldsymbol{G} l+j \omega \boldsymbol{C l}
\end{aligned}
$$

thus allowing the computation of the input admittance matrix $\boldsymbol{Y}^{\prime}=\boldsymbol{I}^{\prime} \boldsymbol{V}^{\prime-1}$

$$
\begin{aligned}
& \Gamma^{\text {bodies }}=\operatorname{sqrtm}\left(\boldsymbol{Y}_{\boldsymbol{E}}^{\text {bodies }} \cdot \boldsymbol{Z}_{M}^{\text {bodies }}\right) \\
& \boldsymbol{Z}_{\boldsymbol{C}}^{\text {bodies }}=\operatorname{sqrtm}\left(\boldsymbol{Y}_{\boldsymbol{E}}^{\text {bodies }^{-1}} \cdot \boldsymbol{Z}_{\boldsymbol{M}}^{\text {bodies }}\right) \\
& \boldsymbol{Y}^{\prime}=\left[\operatorname{sinhm}\left(\boldsymbol{\Gamma}^{\text {bodies }}\right) \cdot \boldsymbol{Z}_{\boldsymbol{C}}^{\text {bodies }^{-1}} \cdot \boldsymbol{Y}^{-1}+\operatorname{coshm}\left(\boldsymbol{\Gamma}^{\text {bodies }}\right)\right] \\
& \cdot\left[\boldsymbol{Y}_{\boldsymbol{E}}^{\text {bodies }^{-1}} \cdot \operatorname{coshm}\left(\boldsymbol{\Gamma}^{\text {bodies }}\right) \boldsymbol{Y}_{\boldsymbol{E}}^{\text {bodies }} \cdot \boldsymbol{Y}^{-1}\right. \\
& \left.+Z_{C}^{b o d i e s} \cdot \operatorname{sinhm}\left(\Gamma^{\text {bodies }}\right)\right]^{-1} \text {. }
\end{aligned}
$$

The previous formula has been derived from [10].

3) Lids Contribution: Since the number of TSCs connected in parallel by the two lids can reach several thousands, very special care has to be taken of the computation of the contribution of that stage. The classical full-wave approach leads to prohibitive computing time or even unsolvable problems, and simulations must be performed with quasi-static solvers. 


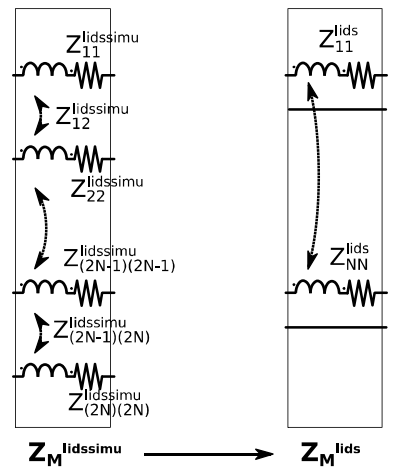

Fig. 6. Illustration of impedance matrix reduction from raw simulation results.

The lids have been modeled with Ansys Q3D quasi-static 3-D electromagnetic solver. In this approach, magnetic and electrical fields are computed separately, and the proposed method provides the following:

1) a $2 N \times 2 N$ "magnetic impedance matrix" $\boldsymbol{Z}_{M}^{\text {lidssimu }}$ that corresponds to the impedance of the lids from the contact points to the $2 N$ conductors of the TSC ( $N$ inner and $N$ outer);

2) a $2 \times 2$ "electrical admittance matrix" $\boldsymbol{Y}_{\boldsymbol{E}}^{\text {lidssimu }}$ that corresponds to the admittance between the two lids.

The raw simulation matrix $Z_{M}^{\text {lidssimu }}$ (of size $2 N \times 2 N$ ) must be reduced to $Z_{M}^{\text {lids }}$ (of size $N \times N$ ) thanks to the following relation (illustrated in Fig. 6):

$$
\begin{aligned}
& \forall i, j \in\{1, \ldots, N\} \\
& Z_{M_{i j}}^{\text {lids }}=Z_{(2 i-1)(2 j-1)}^{\text {lidssimu }}+Z_{(2 i)(2 j)}^{\text {lidssimu }}-Z_{(2 i-1)(2 j)}^{\text {lidssimu }}-Z_{(2 i)(2 j-1)}^{\text {lidssimu }} .
\end{aligned}
$$

The raw admittance matrix $\boldsymbol{Y}_{\boldsymbol{E}}^{\text {lidssimu }}$ (of size $2 \times 2$ ) must be reduced, assuming that the computed coupling admittance between the two lid plates can be evenly distributed among all TSC contact points. As there are $N$ TSCs, an $N \times N$ admittance matrix $\boldsymbol{Y}_{\boldsymbol{E}}^{\text {lids }}$ is built. Furthermore, since the two metallic plates are very close one from another, the $N$ subparts are supposed to be in total influence. $\boldsymbol{Y}_{\boldsymbol{E}}^{\text {lids }}$ is thus a diagonal matrix with equal diagonal coefficients

$$
\boldsymbol{Y}_{\boldsymbol{E}}^{\text {lids }}=-\frac{Y_{E_{12}}^{\text {lidssimu }}+Y_{E_{21}}^{\text {lidssimu }}}{2 N} \boldsymbol{I}_{N} .
$$

Given $\boldsymbol{Z}_{\boldsymbol{M}}^{\text {lids }}$ and $\boldsymbol{Y}_{\boldsymbol{E}}^{\text {lids }}$, one can compute the input admittance $\boldsymbol{Y}^{\prime \prime}=\boldsymbol{I}^{\prime \prime} \boldsymbol{V}^{\prime \prime-1}$ as follows:

$$
\begin{aligned}
\Gamma^{l i d s}= & \operatorname{sqrtm}\left(\boldsymbol{Y}_{\boldsymbol{E}}^{\text {lids }} \boldsymbol{Z}_{\boldsymbol{M}}^{\text {lids }}\right) \\
\boldsymbol{Z}_{\boldsymbol{C}}^{\text {lids }}= & \operatorname{sqrtm}\left(\boldsymbol{Y}_{\boldsymbol{E}}^{\text {lids }}{ }^{-1} \boldsymbol{Z}_{\boldsymbol{M}}^{\text {lids }}\right) \\
\boldsymbol{Y}^{\prime \prime}= & {\left[\operatorname{sinhm}\left(\boldsymbol{\Gamma}^{\text {lids }}\right) \cdot \boldsymbol{Z}_{\boldsymbol{C}}^{\text {lids }}{ }^{-1} \cdot \boldsymbol{Y}^{\prime-1}+\operatorname{coshm}\left(\boldsymbol{\Gamma}^{\text {lids }}\right)\right] } \\
& \cdot\left[\boldsymbol{Y}_{\boldsymbol{E}}^{\text {lids }}{ }^{-1} \cdot \operatorname{coshm}\left(\boldsymbol{\Gamma}^{\text {lids }}\right) \cdot \boldsymbol{Y}_{\boldsymbol{E}}^{\text {lids }} \cdot \boldsymbol{Y}^{\prime-1}\right. \\
& \left.+\boldsymbol{Z}_{\boldsymbol{C}}^{\text {lids }} \cdot \operatorname{sinhm}\left(\boldsymbol{\Gamma}^{\text {lids }}\right)\right]^{-1} .
\end{aligned}
$$

4) Equivalent Impedance: At this step, all the ports of the lids input plane are connected together in parallel. One defines the incidence matrix $\boldsymbol{P}$

$$
\boldsymbol{P}=[1 \cdots 1]
$$

(a)

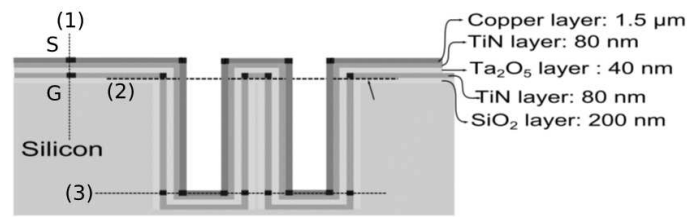

(b)

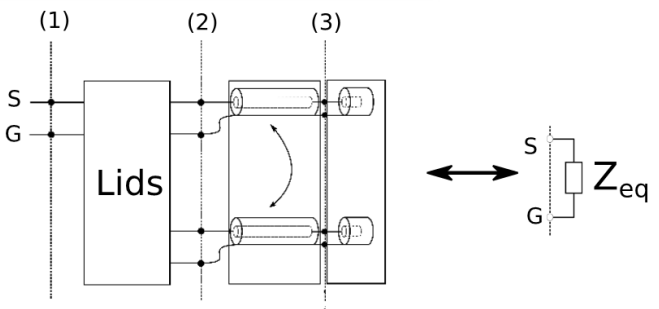

Fig. 7. (a) Cross section of measured radial TSC devices. (b) Equivalent electrical circuit.
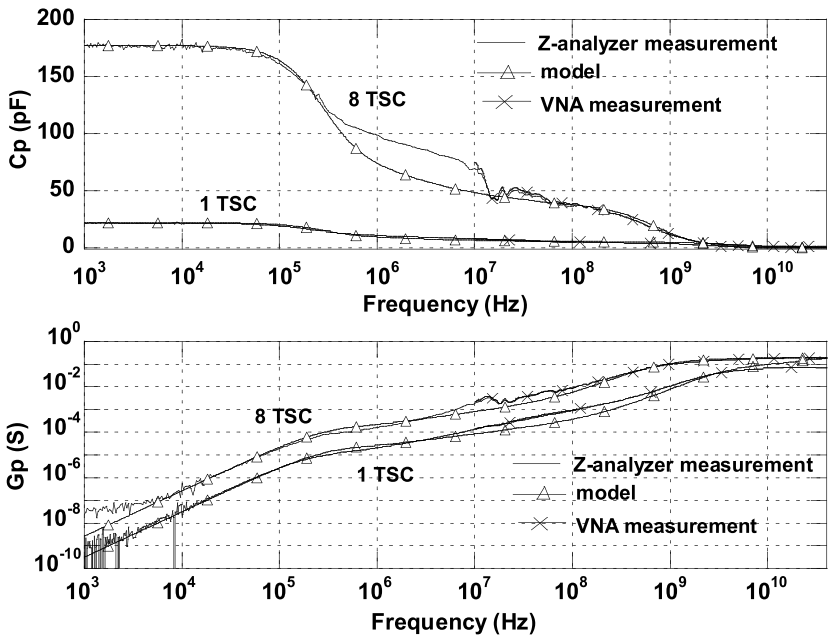

Fig. 8. Measured and modeled impedance response of radial TSC components.

and the equivalent scalar impedance of the whole system $Z_{\text {eq }}=V^{\prime \prime \prime} \cdot I^{\prime \prime \prime-1}$ can finally be written as

$$
Z_{\text {eq }}=\left(\boldsymbol{P} \cdot \boldsymbol{Y}^{\prime \prime} \cdot \boldsymbol{P}^{T}\right)^{-1} .
$$

5) Experimental Validation: The previously presented modeling method for radial TSC has been experimentally validated by measurements from $1 \mathrm{kHz}$ to $40 \mathrm{GHz}$. Large matrices of radial TSC $(N>100$ TSCs $)$ realized with a first generation of MIM stack (TiN $200 \mathrm{~nm} / \mathrm{Al}_{2} \mathrm{O}_{3} 20 \mathrm{~nm} /$ TiN $200 \mathrm{~nm}$ ) had been measured and modeled in [12]. New prototypes realized with a second generation of MIM stack (TiN $80 \mathrm{~nm} / \mathrm{Ta}_{2} \mathrm{O}_{5} 40 \mathrm{~nm} / \mathrm{TiN} 80 \mathrm{~nm} / \mathrm{Cu} 1.5 \mu \mathrm{m}$ ) have been realized and measured [13]. A cross section of such components is presented in Fig. 7.

The components have been characterized from $1 \mathrm{kHz}$ to $110 \mathrm{MHz}$ using Agilent 4294A impedance analyzer and from $10 \mathrm{MHz}$ to $40 \mathrm{GHz}$ with Agilent N5247A PNA-X vector network analyzer. Fig. 8 shows measurement and modeling results of two small components with 1 and 8 TSCs presented as equivalent parallel capacitance $C_{p}=\operatorname{imag}\left(Z(f)^{-1} / 2 \pi f\right)$ and conductance $G_{p}(f)=\operatorname{real}\left(Z(f)^{-1}\right)$.

The low-frequency part of $C_{p}(f)$ gives the capacitance of the component. A first cutoff frequency occurs at $100 \mathrm{kHz}$ 


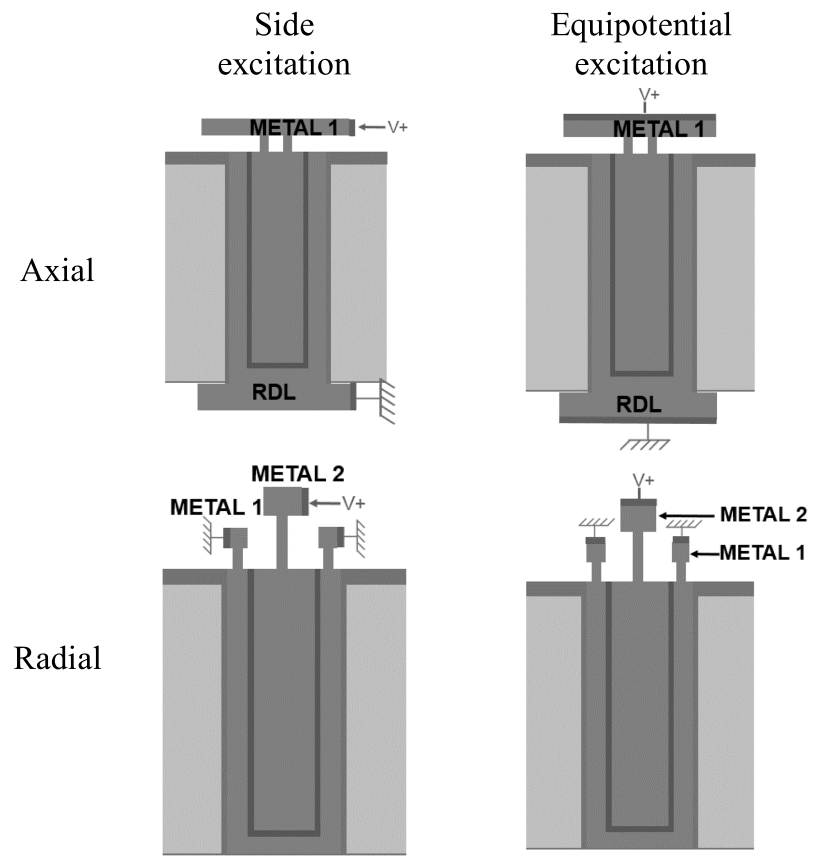

Fig. 9. Four studied configurations of TSC: axial or radial structure and side or equipotential excitation.

due to a high equivalent serial resistance (ESR). The lack of copper layer in the bottom electrode of the measured prototypes is responsible for this performance limitation. Indeed, in that generation of components, only low-conductivity TiN $\left(\sigma_{\mathrm{TiN}}=460 \mathrm{kS} \cdot \mathrm{m}^{-1}\right)$ has been deposited. However, the final components, including copper in each electrode stack, will be drastically enhanced. $C_{p}$ and $G_{p}$ curves show very good agreement between models and measurements on the whole frequency range, except the underestimation of $C p$ in midrange frequency, which is attributed to the not-well-known resistivity of TiN inside the TSC bodies ([9] presents the technological process and describes the nonconformal densification process of TiN, resulting in an inhomogeneous electrical conductivity). These measurements validate the modeling method for the taking into account of electromagnetics effects in the TSC components.

\section{Comparative Study of TSC ARchitectures}

The two modeling methods presented in Section III have been used to perform simulations of realistic, applicationoriented components. The objective of this paper is to point out clear design rules based on electrical performance optimization. The designer will then have to make tradeoffs between electrical performances and technological feasibility. One has considered four integration possibilities for TSC components that are presented in Fig. 9. They are a combination of: 1) axial or radial architecture, and 2) side or equipotential excitation.

For each of the four cases, matrices of 64 and 100 TSCs have been modeled, leading to 8 comparable frequency responses. The MIM stack consists in a 40-nm-thick $\mathrm{Ta}_{2} \mathrm{O}_{5}$ layer between copper electrodes. In axial TSC architecture, the front-side metal 1 (M1) layer is $150 \mathrm{~nm}$ thick, and the back-side redistribution layer is $7 \mu \mathrm{m}$ thick. In radial

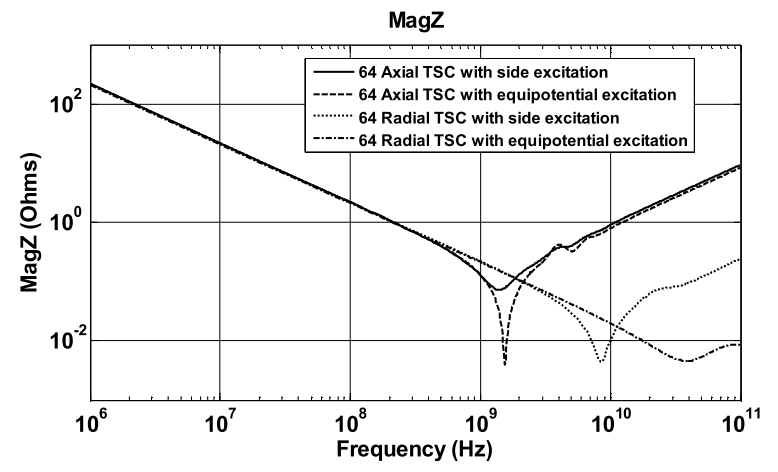

Fig. 10. Simulated impedance magnitude frequency response of 64 TSC.

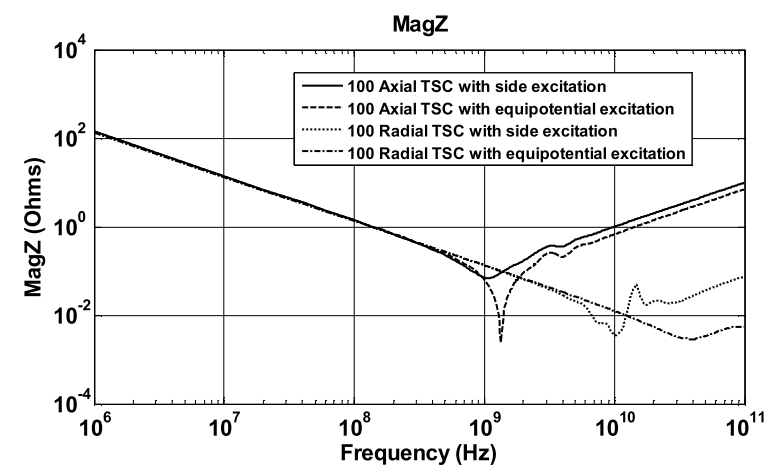

Fig. 11. Simulated impedance magnitude frequency response of 100 TSC.

TABLE I

ELECTRICAL DATA OF RADIAL EQUIPOTENTIAL TSC COMPONENTS

\begin{tabular}{|c|c|c|c|c|c|c|c|c|}
\hline 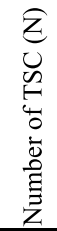 & 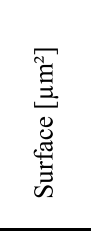 & 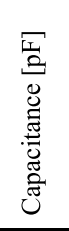 & 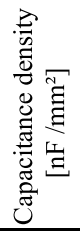 & $\begin{array}{l}\widetilde{\mathcal{C}} \\
\underline{\Xi} \\
\widetilde{\sim} \\
\tilde{\sim}\end{array}$ & 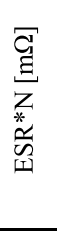 & 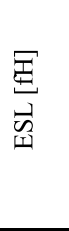 & 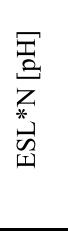 & 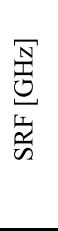 \\
\hline 1 & 285 & 11.8 & 41.4 & 296 & 296 & 1349 & 1349 & 39.9 \\
\hline 2 & 623 & 23.6 & 37.9 & 147 & 294 & 672 & 1344 & 39.9 \\
\hline 64 & 22842 & 730 & 31.9 & 4.50 & 288 & 20 & 1280 & 39.7 \\
\hline 100 & 35765 & 1180 & 33.0 & 2.92 & 292 & 13.5 & 1350 & 39.6 \\
\hline 256 & 90506 & 3008 & 33.2 & 1.14 & 292 & 5.1 & 1305 & 39.6 \\
\hline 400 & 141138 & 4689 & 33.2 & 0.73 & 292 & 3.4 & 1360 & 39.6 \\
\hline
\end{tabular}

architectures, M1 and M2 are, respectively, 150 and $340 \mathrm{~nm}$ thick. Each 64 TSC component is $730 \mathrm{pF}$ for $0.022 \mathrm{~mm}^{2}$, and each 100 TSC component is $1.18 \mathrm{nF}$ for $0.035 \mathrm{~mm}^{2}$. These values correspond to a $33-34 \mathrm{nF} / \mathrm{mm}^{2}$ capacitance density in both cases. As demonstrated in [5], the maximum capacitance density obtainable with planar MIM capacitors for PDN decoupling capacitors, considering the breakdown voltage and the dielectric permittivity of available materials, is also $30 \mathrm{nF} / \mathrm{mm}^{2}$, which is comparable to the performances of the TSC presented in this paper.

Figs. 10 and 11, respectively, plot the impedance magnitude responses of 64 and 100 TSC matrices.

Radial architectures provide a one-decade-higher SRF compared to axial architectures, whether the excitation is on side or equipotential. This result is due to the coaxial configuration of the radial architecture that minimizes the overall 
inductance on the current path, thus reducing the equivalent serial inductance (ESL) and then increasing the SRF. As the maximum decoupling frequency strongly depends on a high SRF, radial architectures should be preferred for applications around $4 \mathrm{GHz}$ and higher.

The equipotential excitation is also preferable over the side excitation because the current path in lids is reduced, leading to the reduction of the ESL and of the ESR. The impact of ESL reduction is more visible on the radial architecture because the coaxial bodies have a little contribution. In that case, the SRF is increased by almost another decade.

Finally, the comparative study leads to the election of the radial architecture with equipotential excitation as the best candidate decoupling capacitors integration with TSC. The radial architecture with equipotential excitation has then been further investigated. Matrices of 1, 2, 64, 100, 256, and 400 TSCs have been modeled, and electrical data have been extracted and are reported in Table I.

The mean capacitance density is about $35 \mathrm{nF} / \mathrm{mm}^{2}$. This value is a good middle point between planar MIM capacitors (around $5 \mathrm{nF} / \mathrm{mm}^{2}$ [14]) and optimized double trenched capacitors (which can reach several hundred $\mathrm{nF} / \mathrm{mm}^{2}$ [15], [16]). The main advantage of this technology is then the cointegration of high-value capacitors with the standard TSV process in interposers.

The ESR and ESL values multiplied by the number $N$ of TSCs are almost constant values, indicating that the coaxial bodies are responsible for them: As all coaxial bodies are connected in parallel, ESR and ESL are inversely proportional to $N$. In the case of $N=400$ TSCs, the ESR is $0.73 \mathrm{~m} \Omega$ and the ESL is $3.4 \mathrm{fH}$, for a capacitance of $4.6 \mathrm{nF}$. The low ESR value has been enabled by the use of thick copper electrodes, while the radial topology allows low ESL. Finally, the SRF is almost a constant value of $39 \mathrm{GHz}$, which is due to the inverse proportionality relationship between the capacitance value $C$ and the ESL (as $N$ increases, $C$ increases and ESL decreases). This high-value SRF even for large matrices of TSC is also a promising result for TSC integration.

\section{CONCLUSION}

Two powerful predictive modeling methods for axial and radial architectures of TSC have been proposed and validated. Measurements of radial TSC have been reported on $\mathrm{Ta}_{2} \mathrm{O}_{5}$ radial components, and simulations of various TSC configurations, with axial or radial architecture, with side or equipotential excitation of the electrodes and with up to 400 TSC have been performed. The final comparative study concludes to the election of radial architecture with equipotential excitation for TSC components integration. The electrical performances of the modeled components are very promising for decoupling applications in power delivery networks.

\section{REFERENCES}

[1] R. T. Rao and S. Madhavan, Introduction to System-on-Package (SoP) Miniaturization of the Entire System. New York, NY, USA: McGraw-Hill, 2008.
[2] H. Lee, Y. S. Choi, E. Song, K. Choi, T. Cho, and S. Kang, "Power delivery network design for 3D SIP integrated over silicon interposer platform," in Proc. 57th IEEE Electron. Compon. Technol. Conf. (ECTC), Sparks, NV, USA, May/Jun. 2007, pp. 1193-1198.

[3] A. Takano, M. Sunohara, M. Higashi, I. Hayakawa, K.-I. Ohta, and Y. A. Sasajima, "Development of Si interposer with low inductance decoupling capacitor," in Proc. 61st IEEE Electron. Compon. Technol. Conf. (ECTC), Lake Buena Vista, FL, USA, May/Jun. 2011, pp. 849-854.

[4] L. Wan et al., "Embedded decoupling capacitor performance in high speed circuits," in Proc. 55th IEEE Electron. Compon. Technol. Conf. (ECTC), Lake Buena Vista, FL, USA, May/Jun. 2005, pp. 1617-1622.

[5] Y. Wang, S. Xiang, P. M. Raj, H. Sharma, B. Williams, and R. Tummala, "Solution-derived electrodes and dielectrics for low-cost and highcapacitance trench and through-silicon-via (TSV) capacitors," in Proc. 61st IEEE Electron. Compon. Technol. Conf. (ECTC), Lake Buena Vista, FL, USA, May/Jun. 2011, pp. 1987-1991.

[6] O. Guiller, S. Joblot, Y. Lamy, A. Farcy, E. Defay, and K. Dieng, "Through silicon capacitor co-integrated with TSV as an efficient 3D decoupling capacitor solution for power management on silicon interposer," in Proc. 64th IEEE Electron. Compon. Technol. Conf. (ECTC), Orlando, FL, USA, May 2014, pp. 1296-1302.

[7] K. Dieng et al., "Through silicon capacitors (TSC) for noise reduction in power distribution network," in Proc. 65th IEEE Electron. Compon. Technol. Conf. (ECTC), San Diego, CA, USA, May 2015, pp. 247-253.

[8] K. Dieng et al., "Electrical model of different architectures of through silicon capacitors for high frequency power distribution network (PDN) decoupling operations," in Proc. 66th IEEE Electron. Compon. Technol. Conf. (ECTC), Las Vegas, NV, USA, Jun. 2016, pp. 74-81.

[9] O. Guiller, S. Joblot, Y. Lamy, A. Farcy, J. F. Carpentier, and E. Defay, "Through silicon capacitor co-integrated with TSVs on silicon interposer," Microelectron. Eng., vol. 120, pp. 121-126, May 2014.

[10] A. A. Bhatti, "A computer based method for computing the $\mathrm{N}$-dimensional generalized ABCD parameter matrices of $\mathrm{N}$-dimensional systems with distributed parameters," in Proc. 22nd Southeastern Symp. Syst. Theory, Mar. 1990, pp. 590-593.

[11] D. A. Frickey, "Conversions between S, Z, Y, H, ABCD, and T parameters which are valid for complex source and load impedances," IEEE Trans. Microw. Theory Techn., vol. 42, no. 2, pp. 205-211, Feb. 1994.

[12] K. Dieng et al., "Electrical model and characterization of through silicon capacitors (TSC) in silicon interposer," in Proc. IEEE Int. 3D Syst. Integr. Conf. (DIC), Cork, Ireland, 2014, pp. 1-8.

[13] K. Dieng et al., "Electrical model and characterization of through silicon capacitors (TSC) in silicon interposer," in Proc. 20th IEEE Workshop Signal Power Integr. (SPI), Turin, Italy, Dec. 2016, pp. 1-4.

[14] A. Bajolet et al., "Three-dimensional $35 \mathrm{nF} / \mathrm{mm}^{2}$ MIM capacitors integrated in BiCMOS technology," in Proc. 35th Eur. Solid-State Device Res. Conf. (ESSDERC), Grenoble, France, Sep. 2005, pp. 121-124.

[15] F. Lallemand and F. Voiron, "Silicon interposers with integrated passive devices, an excellent alternativ to discrete components," in Proc. IEEE Eur. Microelectron. Packag. Conf. (EMPC), Sep. 2013, pp. 1-6.

[16] J. H. Klootwijk et al., "Ultrahigh capacitance density for multiple ALDgrown MIM capacitor stacks in 3-D silicon," IEEE Electron Device Lett., vol. 29 , no. 7 , pp. 740-742, Jul. 2008.

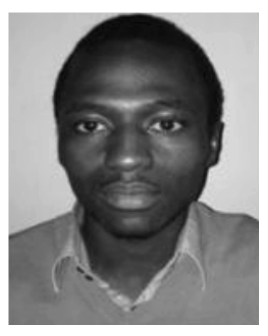

Khadim Dieng received the B.Eng. and M.Sc. degrees in microelectronics and telecommunications from Polytech Marseille, Marseille, France, in 2013. He is currently pursuing the Ph.D. degree with the Institute of Microelectronics, Electromagnetism, and Photonics, Institut Polytechnique de Grenoble, Grenoble, France.

His current research interests include a new decoupling solution for power distribution network of 3-D integrated circuit and through silicon capacitors. 


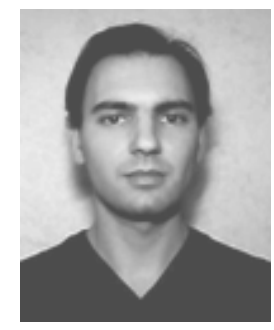

Philippe Artillan received the B.Eng. and M.Sc. degrees in microelectronics from the Institut National des Sciences Appliquées, Toulouse, France, in 2005, and the Ph.D. degree from the Université de Toulouse, Toulouse, in 2008.

$\mathrm{He}$ was an Associate Professor with the Radio Frequency and Millimeter-Wave Group, Characterization and Microwave Laboratory, University of Savoie Mont Blanc, Chambéry, France. His current research interests include integrated passive components for 3-D integration technologies, and particularly in electromagnetic modeling, broadband characterization, and material parameters extraction.

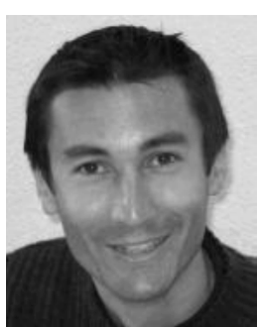

Cédric Bermond received the Ph.D. degree in electronic, optronic, and systems from the University of Savoie, Chambéry, France, in 2001.

$\mathrm{He}$ is currently an Assistant Professor at the Characterization and Microwave Laboratory, University of Savoie Mont Blanc, Chambéry. His current research interests include materials characterization for integrated components, microwave characterization, and modeling and design of interconnects and passive components in advanced integrated circuits.

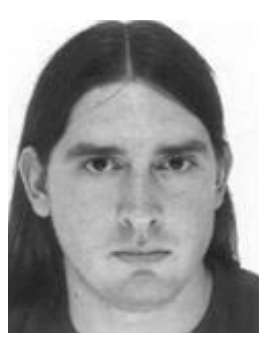

Grégory Houzet received the M.Sc. and $\mathrm{Ph} . \mathrm{D}$. degrees from Lille University, Lille, France, in 2009 .

$\mathrm{He}$ is currently an Assistant Professor with the Laboratory of High Frequency and Characterization, University of Savoie, Chambéry, France. His current research interests include microwave material characterization for 3-D integrated components.

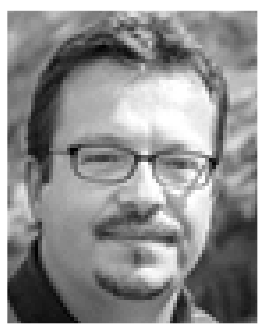

Alexis Farcy received the bachelor's degree in electronic engineering from the "Institut des Sciences et Techniques de Grenoble," Grenoble, France, in 2000, and the Ph.D. degree in electronic, optronic, and systems from the University of Savoie, Chambéry, France, in 2009.

He was with STMicroelectronics, Crolles, France. From 2000 to 2007, he was involved in the Advanced Interconnects and Passive Components Module. Since 2007, he has been involved in the 3-D integration field on innovative technologies, processes, and materials for 3-D integration, 3-D performance assessment, and 3-D technologies for photonics.

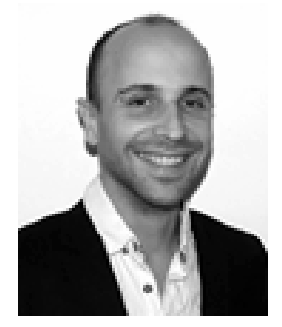

Olivier Guiller received the B.Sc. degree in physics and astrophysics from Nottingham Trent University, Nottingham, U.K., in 2008, the M.Sc. degree in materials science from Rennes University, Rennes, France, in 2009, and the Ph.D. degree in nanoelectronics and nanotechnologies from Grenoble Alpes University, Gières, France, in 2015.

In 2015, he joined Circuits Multi-Projets (CMP), Grenoble, France, and was involved in 3-D integration and advanced packaging subjects.

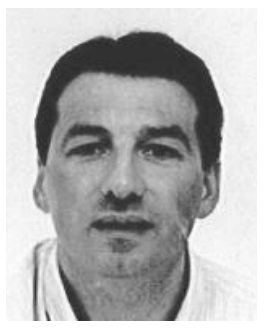

Thierry Lacrevaz received the Ph.D. degree in electronic, optronic, and systems from the University of Savoie, Chambéry, France, in 2005.

$\mathrm{He}$ is currently an Assistant Professor with the Characterization and Microwave Laboratory, University of Savoie Mont Blanc, Chambéry. His current research interests include microwave characterization and particularly in wideband frequency characterization of materials for integrated components, modeling, and design of passive components in advanced integrated circuits.

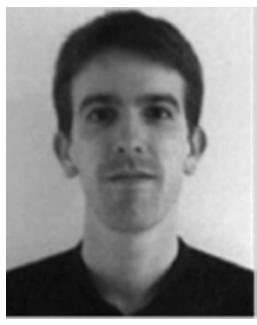

Sylvain Joblot received the materials and science engineering degree from the "Institut National des Sciences Appliquées de Lyon," Lyon, France, in 2003, and the Ph.D. degree from the University of Nice Sophia-Antipolis, Nice, France, in 2007.

He was with STMicroelectrics, Crolles, France, and CNRS-CRHEA, Valbonne, France, where he was involved in the epitaxial growth of GaN-based structures on silicon (001) substrate. In 2013, he joined the Process Development Group, STMicroelectronics, and FEOL Research and Development Team, where he was in charge of the ion implantation process developments. $\mathrm{He}$ has authored and coauthored 32 publications and 14 published patents.

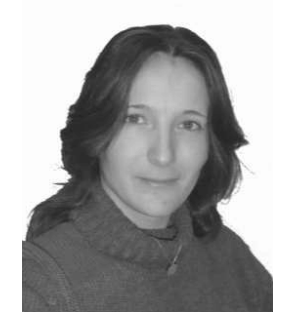

Anne-Laure Perrier received the Ph.D. degree from the Laboratory of Microwaves and Characterization, Université Savoie Mont Blanc, Chambéry, France, in 2006 .

She is currently an Assistant Professor with the Characterization and Microwave Laboratory, University of Savoie Mont Blanc. She designs and realizes RF sensors for magnetic resonance imaging applications. Her current research interests include microwave theory and characterization.

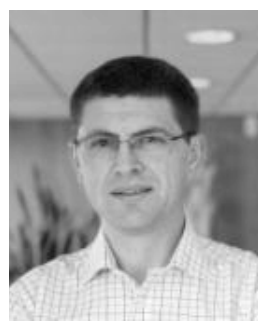

Yann Lamy received the bachelor's degree in solid state physics from ESPCI Paris-Tech, Paris, France, and Paris XI University, Orsay, France, in 2003, and the Ph.D. degree in microwave and optical communications engineering, in 2006.

Since 2012, he has been a Program Manager with the Silicon Component Division of LETI, Grenoble, France. His current research interests include silicon processing, advanced 3-D packaging, millimeterwave devices, and integrated passives.

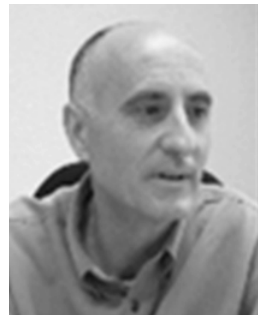

Bernard Fléchet received the B.S. and M.S. degrees in applied physics from University Joseph Fourier Grenoble, France, and the Ph.D. degree in optic, optoelectronic, and microwave from the National Polytechnic Institute of Grenoble, Grenoble, in 1991.

$\mathrm{He}$ is currently a Professor with the Characterization and Microwave Laboratory and the Director of the Electrical Engineering Department, University of Savoie, Chambéry, France. His current research interests include modeling and design of interconnects and passive components in advanced integrated circuits. 